\title{
VIBRATION GENERATOR DEVICE BASED ON INDUSTRIAL VIBRATORS
}

\author{
PÉTER DECSI *1 AND ISTVÁN SZALAI ${ }^{1}$ \\ ${ }^{1}$ Institute of Mechatronics Engineering and Research, University of Pannonia, Gasparich Márk utca 18/A, \\ Zalaegerszeg, 8900, HUNGARY
}

\begin{abstract}
A low-cost vibration generator device based on industrial vibrators was designed. The control software was implemented in LabVIEW Environment. The device is able to generate an oscillating force of $8 \mathrm{kN}$ and an amplitude of up to $4 \mathrm{~mm}$ at a frequency of $50 \mathrm{~Hz}$ to model low-amplitude, high-frequency vehicle vibrations. A National Instruments myRIO device was responsible for data acquisition, with which a signal of a piezoelectric accelerometer was detected. The test results show that the device is able to generate a sinusoidal harmonic acceleration.
\end{abstract}

Keywords: industrial vibrator, vibration generator, test equipment

\section{Introduction}

The suspension of a car acts as a connector between the chassis and the road. It provides a comfortable ride for passengers while maintaining maneuverability. Nowadays, comfort is a prerequisite so car manufacturers have started developing new technologies for isolating vibrations $[1,2]$. The vibration can be dampened or converted into electrical energy [3]. Electro- [4] and magnetorheological dampers [5,6] are filled with a special fluid. Small ferromagnetic particles are dispersed in a carrier fluid, usually silicone oil. The diameters of the particles fall within the micro- and nanometre ranges. When subjected to an electric or magnetic field, the particles form chains. These chains provide an elevated level of resistance against shear stress, therefore, the apparent viscosity of the fluid rises $[7,8]$.

Using adjustable shock absorbers, the natural frequency of the suspension can be altered. The damping coefficient can be varied depending on the road conditions or the driver's preference. The reaction time of these fluids is very short (approximately $10 \mathrm{~ms}$ ), so the technology can be used in systems where short reaction times are required [9].

Suspension-testing equipment is crucial during the development phase. The acquisition cost of appliances for this task is very high. Our goal was to develop a low-cost piece of equipment for testing shock absorbers that is capable of generating high frequencies (within an acceptable range for vehicles) and vibrations of low amplitude.

\footnotetext{
*Correspondence: decsi.peter@mk.uni-pannon.hu
}

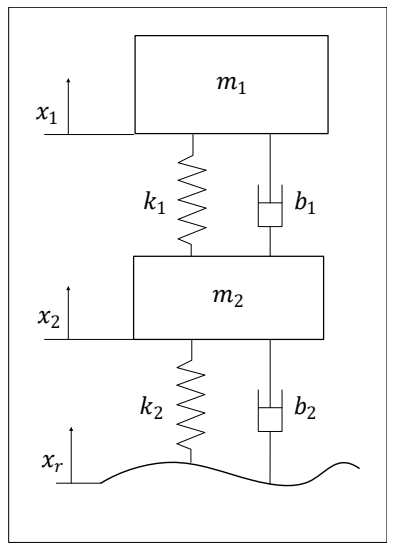

(a) structure model

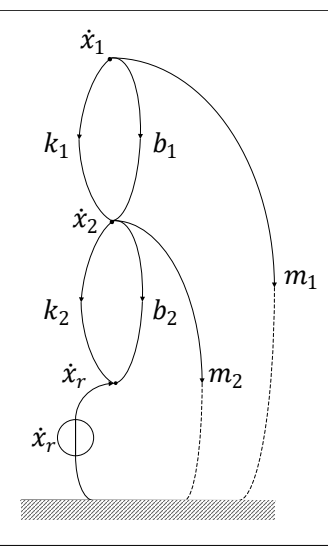

(b) structure graph
Figure 1: Quarter-car model

\section{Modeling the vehicle}

Several options of modeling the suspension of vehicles are available; quarter-, half- and full-car models can be used to describe the system, which is chosen depends on the aim of the study. The quarter-car model was chosen for this study due to its simplicity. Industrial vibrators are able to generate vibrations of constant amplitude. To change the generated force and amplitude, it is necessary to stop the device and mechanically set the eccentricity, therefore, the roll, pitch and yaw of the vehicle is hard to model with such a device. Fig. 1 shows a quarter-car model. The system has two degrees of freedom (DOF), $m_{1}$ denotes the mass of the chassis, $m_{2}$ represents the mass of the wheel, which is usually referred to as the unsprung mass. The related displacements are labelled as $x_{1}$ 
and $x_{2}$, spring stiffnesses as $k$ and viscous damping coefficients as $b$. The wheel and chassis are connected through a spring $k_{1}$ and a damper $b_{1}$. The wheel and the road are connected through a spring and damper (elastic tire with damping loss) $k_{2}, b_{2}$. Based on the structure graph, the equilibrium equations can be written in the form:

$$
\begin{aligned}
m_{1} \ddot{x}_{1}= & -b_{1}\left(\dot{x}_{1}-\dot{x}_{2}\right)-k_{1}\left(x_{1}-x_{2}\right) \\
m_{2} \ddot{x}_{2}= & b_{1}\left(\dot{x}_{1}-\dot{x}_{2}\right)+k_{1}\left(x_{1}-x_{2}\right)+ \\
& +b_{2}\left(\dot{x}_{r}-\dot{x}_{2}\right)+k_{2}\left(x_{r}-x_{2}\right) .
\end{aligned}
$$

Based on Fig. 1 and Eq. 1, it is clear that the system has a cross-variable source, namely speed.

\section{Modeling the proposed system}

This research presents an equivalent model based on industrial vibrators. Industrial vibrators are traditional asynchronous motors with a dual shaft on which eccentric masses are mounted.

$$
f_{c}=m r \omega^{2}
$$

where $m$ denotes the eccentric mass, $r$ represents the eccentricity, which is the distance between the axis center point and the center of gravity of the eccentric mass, and $\omega$ stands for the rotational speed. Due to the eccentricity, a centrifugal force is generated because of the rotation (Eq. 2). If two motors of opposite rotational directions are mounted together, lateral forces cancel each other out, therefore, a one-axis oscillation is created. A system with two degrees of freedom was designed using industrial vibrators. The model of the system is depicted in Fig. 2: The differential equations of this system are:

$$
\begin{aligned}
m_{1} \ddot{x}_{1} & +k_{1}\left(x_{1}-x_{2}\right)+ \\
& +b_{1}\left(\dot{x}_{1}-\dot{x}_{2}\right)+f_{\text {in }}=0 \\
m_{2} \ddot{x}_{2} & -k_{1}\left(x_{1}-x_{2}\right)- \\
& -b_{1}\left(\dot{x}_{1}-\dot{x}_{2}\right)+k_{2} x_{2}+\dot{x}_{2} b_{2}=0
\end{aligned}
$$

According to the impedance network of the quartercar model shown in Fig. 3, the transfer function can be written in the form Eq. 4. The impedance network of the proposed system is shown in Fig. 4 and contains the transfer function of Eq. 5:

It can be seen that the transfer function $X_{1} / X_{r}$ of the quarter-car model (Eq. 4) is similar to that of the transfer

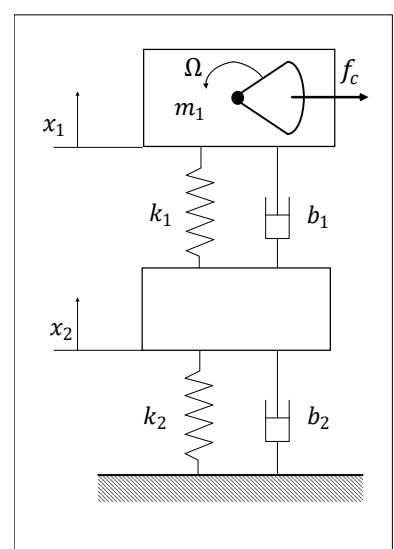

(a) structure model

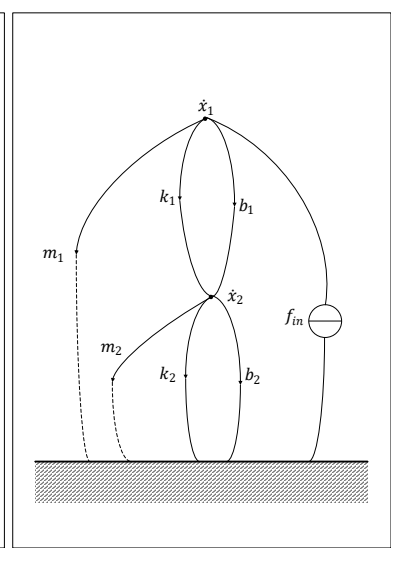

(b) structure graph

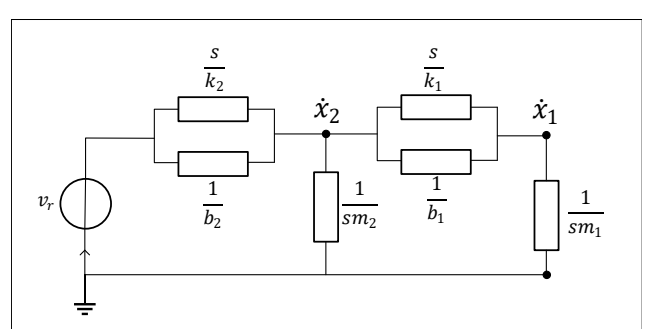

Figure 3: Impedance network of the quarter-car model

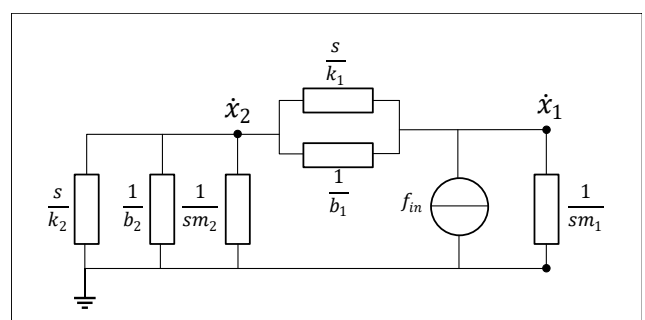

Figure 4: Impedance network of the vibrator model

Figure 2: Vibrator model 
function $X_{1} / F$ of the proposed system (Eq. 5). The characteristic polynomials, i.e. the denominators, are identical as the two systems differ only in terms of the excitation source. The numerators are of the same degree, therefore, the characteristics of the system response are similar, that is to say the dynamics of the two systems are not significantly different. The breakpoints in terms of the frequency response are expected to be shifted.

\section{Implementation}

After deriving the differential equations, a Simulink model was created to choose the optimal combinations of parameters (mass, spring stiffness and damping coefficients). According to the simulations, the connection between the chassis and the ground should be as stiff as possible in order to focus the force on the shock absorbers. It should not be too rigid, otherwise the force acting on the ground would be excessive and affect the building too significantly. The moving mass, where the motors are mounted, should be small to achieve the maximum possible exciting force, therefore, more force can be used to accelerate the payload.

During the design process, models with two or three degrees of freedom were examined. The motion became chaotic with three independent masses, that is 3 DOFs, so the spring stiffness available was insufficient to produce a stable and predictable shape of sinusoidal motion. It was concluded that a system with 2 DOFs generates a stable sinusoidal oscillation with multiple frequency components in terms of the shape of motion.

The device, shown in Fig. 5, consists of two threephase asynchronous two-pole industrial vibrators each

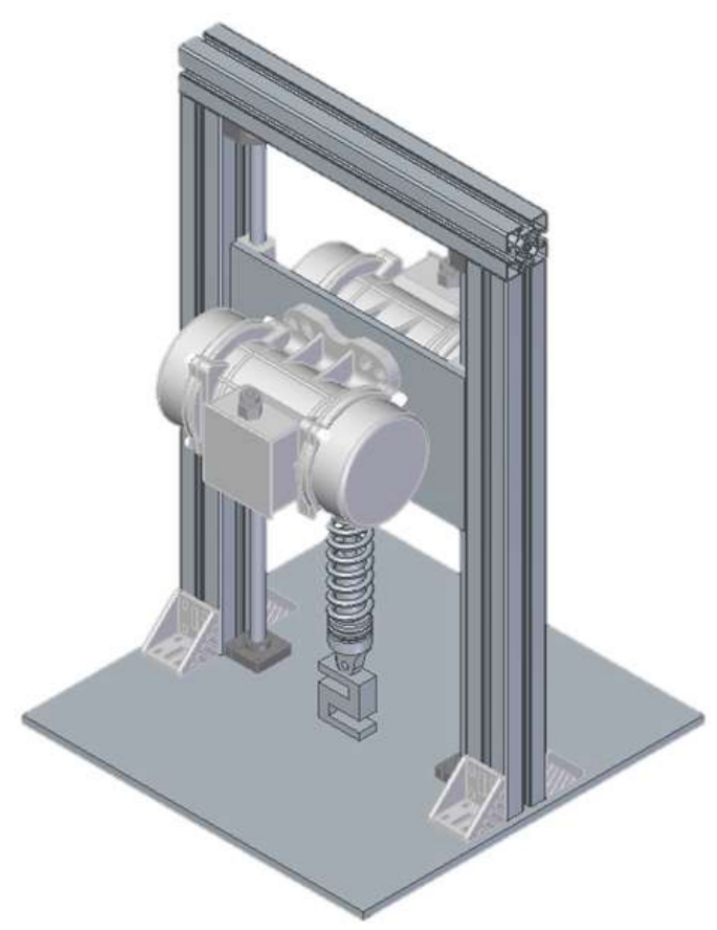

Figure 5: The implemented vibration generator with a nominal performance of $300 \mathrm{~W}$. The centrifugal force can be incrementally set up to $4070 \mathrm{~N}$ at a frequency of $50 \mathrm{~Hz}$ on one motor. The motors are operated with frequency converters, thus the frequency can be set. If the motors operate in opposite rotational directions and a suitable arrangement is applied, the lateral forces cancel each other out, thereby creating a one-axis oscillation. The frequency converters are controlled with analogue signals generated by a National Instruments myRIO device.

A piezoelectric accelerometer was mounted on the sheet holding the vibrating motors. A high-pass filter with a cutoff frequency of $1 \mathrm{~Hz}$ was installed to cancel out the bias voltage of the accelerometer. Data was acquired by the Field-Programmable Gate Array (FPGA) module of the myRIO device on analogue channels. The AC signal was coupled to a high-pass filter with a cutoff frequency of $1 \mathrm{~Hz}$. The sensitivity of the accelerometer was $100 \mathrm{mV} / \mathrm{g}$ and the analogue input of the myRIO device was $\pm 10 \mathrm{~V}$. An instrumentation amplifier with an amplification of 7.08 was built to utilize the full range of the A/D converter. The FPGA module takes a sample according to the previously set sampling frequency, which can be set up to $100 \mathrm{kHz}$. The data is stored temporarily in the First In First Out (FIFO) memory on the myRIO device.

A second Virtual Instrument (VI), which displays and stores the data in Technical Data Management Streaming (.TDMS) file format, was run on the controlling PC. The VI read out data from the FIFO memory in batches. These batches were stored in the TDMS file, creating a reliable data acquisition. Meanwhile, following the application of a Fast Fourier Transform algorithm, a frequency spectrum was displayed on the controlling PC.

\section{Test results}

A test measurement was taken after the implementation. For test purposes, the frequency converters were set at $16 \mathrm{~Hz}$. Fig. 6 shows a long-term test and Fig. 7 shows the measurement of a short-term acceleration. It can be seen that the acceleration is approximately sinusoidal and consists of two main frequency components. Fig. 6 shows that the acceleration was stable over an extended period of time with several protrusions. Fig. 8 shows that the set

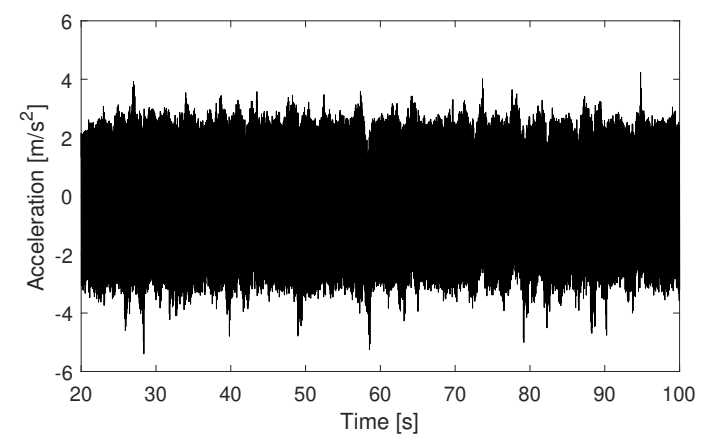

Figure 6: Test measurement, long duration 


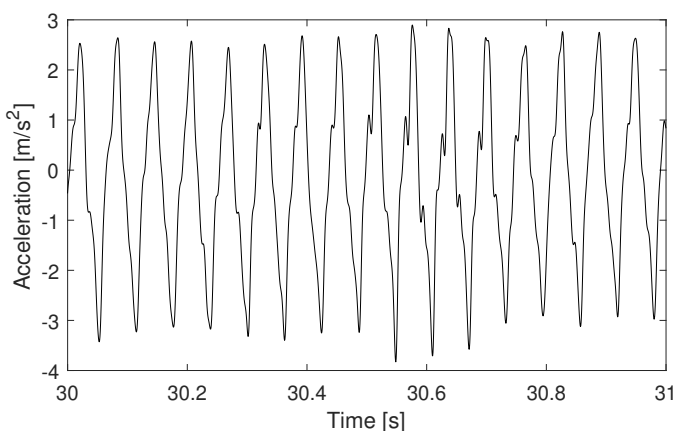

Figure 7: Test measurement, short duration

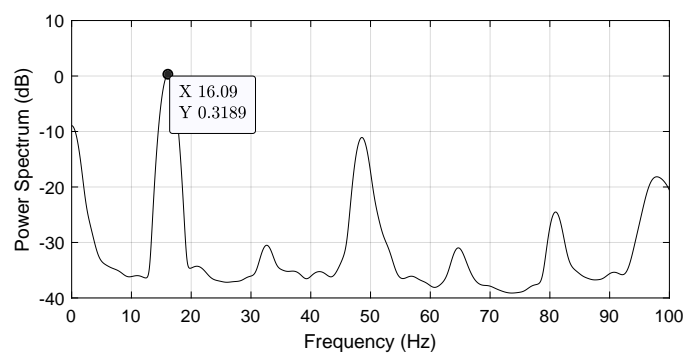

Figure 8: Frequency spectrum of the test measurement

frequency was present in the spectrum alongside multiple frequency components.

\section{Conclusion}

According to our experience and the aforementioned results, it can be concluded that a cost-effective vibration generator was designed and produced. The device is able to generate an amplitude of vibration equal to $4 \mathrm{~mm}$ with a frequency of up to $50 \mathrm{~Hz}$. The total centrifugal force was as high as $8 \mathrm{kN}$, of which $7500 \mathrm{~N}$ could be focused on the examined sample. The frequency can be set between $1 \mathrm{~Hz}$ and $50 \mathrm{~Hz}$, however, as shown in Eq. 2, the centrifugal force depends on the rotational speed.

The amplitude cannot be set, and when the natural frequency of the system, namely $1.3 \mathrm{~Hz}$, is exceeded, the amplitude becomes constant. At this frequency, the centrifugal force is low so resonance can be avoided. The excitation force can be set between 0 and $100 \%$ by modifying the eccentricity of the motors. To set the eccentricity, the motors must be powered down and the side covers removed.

The vibration generators that are typically used (electrodynamic and hydraulic) are able to create higher amplitudes and higher vibrational frequencies, but these devices belong to a different cost category.

\section{Acknowledgement}

The project has been supported by the European Union, co-financed by the European Social Fund. EFOP-3.6.216-2017-00002

\section{REFERENCES}

[1] Qin, Y.; Tang, X.; Jia, T.; Duan, Z.; Zhang, J.; Li, Y.; Zheng, L.: Noise and vibration suppression in hybrid electric vehicles: State of the art and challenges, Renew. Sustain. Energy Rev., 2020, 124, DOI: 10.1016/j.rser.2020.109782

[2] Ning, D.; Sun, S.; Du, H.; Li, W.; Li, W.: Control of a multiple-DOF vehicle seat suspension with roll and vertical vibration, J. Sound Vib., 2018, 435, 170-191, DOI: 10.1016/j.jsv.2018.08.005

[3] Zhang, Z.; Xiang, H.; Shi, Z.; Zhan, J.: Experimental investigation on piezoelectric energy harvesting from vehicle-bridge coupling vibration, $\mathrm{En}$ ergy Convers. Manag., 2018, 163, 169-179, DOI: 10.1016/j.enconman.2018.02.054

[4] Holzmann, K.; Kemmetmüller, W.; Kugi, A.; Stork, M.: Design, mathematical modeling and control of an assymetrical electrorheological damper, IFAC Proceedings Volumes, 2006, 39(16), 372-377, DOI: 10.3182/20060912-3-DE-2911.00066

[5] Graczykowski, C.; Pawłowski, P.: Exact physical model of magnetorheological damper, Appl. Math. Model., 2017, 47, 400-424, DOI: 10.1016/j.apm.2017.02.035

[6] Yao, G.Z.; Yap, F.F.; Chen, G.; Li, W.H.; Yeo, S.H.: MR damper and its application for semiactive control of vehicle suspension system, Mechatronics, 2002, 12(7), 963-973, DOI: 10.1016/S09574158(01)00032-0

[7] Carlson, J.D.: What Makes a Good MR Fluid?, J. Intel. Mat. Syst. Str., 2002, 13(7-8), 431-435, DOI: 10.1106/104538902028221

[8] Rankin, P.J.; Ginder, J.M.; Klingenberg, D.J.: Electro- and magneto-rheology, Curr. Opin. Colloid Interface Sci., 1998, 3(4), 373-381, DOI: 10.1016/S1359-0294(98)80052-6

[9] Olabi, A.; Grunwald, A.: Design and application of magneto-rheological fluid, Mater. Des., 2007, 28(10), 2658-2664, DOI: 10.1016/j.matdes.2006.10.009 